\title{
The Investigation of The Anisotropic Analytical Algorithm (AAA) and the Acuros XB (AXB) Dose Calculation Algorithms Accuracy in Surface and Buildup Region for 6 MV Photon Beam Using Gafchromic EBT3 Film
}

\author{
(1) Nazmiye DÖNMEZ KESEN, (1) Canan KÖKSAL AKBAŞ \\ Department of Radiotherapy, Istanbul University Institute of Oncology, İstanbul-Turkey
}

\begin{abstract}
OBJECTIVE
Due to the complexity of build-up region dosimetry, treatment planning system (TPS) calculations may present significant inaccuracy in surface and buildup regions. The main aim of the current study was to investigate the calculated build-up and surface doses for $6 \mathrm{MV}$ photon beams by Eclipse TPS version 15.1 Anisotropic Analytical Algorithm (AAA) and Acuros XB (AXB) algorithm.

\section{METHODS}

Calculations were compared to surface doses measured by Markus parallel-plate ionization chamber which is admitted asan alternative to the extrapolation chambers to obtain the most accurate results for surface region and by Gafchromic EBT3 film which is frequently preferred in in vivo dosimetry (IVD) applications for skin dose measurements because of their practical usage. The measurements were made for $5 \times 5,10 \times 10$, and $20 \times 20 \mathrm{~cm}^{2}$ field sizes at the surface and in the buildup region of $6 \mathrm{MV}$. Dosimetry systems were placed in a water equivalent solid phantom; all measurements were performed at $100 \mathrm{~cm}$ source-detector distance.
\end{abstract}

\section{RESULTS}

The surface doses using $6 \mathrm{MV}$ photon beams for $5 \times 5,10 \times 10$, and $20 \times 20 \mathrm{~cm}^{2}$ field sizes at $0.07 \mathrm{~mm}$ was found to be $14.00 \%$ and $12.79 \% ; 19.69 \%$ and $18.80 \%$; and $30.87 \%$ and $27.07 \%$ for Markus chamber and Gafchromic EBT3 film, respectively.

\section{CONCLUSION}

In clinics, to be sure of the correctness of surface and buildup region dose calculation, QA procedures must be performed for all algorithms implanted in TPS before using them for patient treatment planning. Since it exhibits the similar results by Markus parallel plate ion chamber in the surface and buildup region, Gafchromic EBT3 film can be a preferable dosimeter in IVD.

Keywords: Acuros XB algorithm; anisotropic analytical algorithm; gafchromic film.

Copyright $\odot$ 2021, Turkish Society for Radiation Oncology

\section{Introduction}

In radiotherapy, surface dosimetry is very complicated issue. The reason of this complication is the rapid dose change between the dose entrance point and the maxi- mum dose depth. Compton interactions are usually the main reason of radiation absorption in tissues. When the primary photon beams interact with a homogenous material, secondary particles such as electrons or positrons are liberated and by the increasing the depth

Dr. Nazmiye DÖNMEZ KESEN

İstanbul Üniversitesi Onkoloji Enstitüsü,

Radyoterapi Anabilim Dalı,

İstanbul-Turkey

E-mail: nazo94@gmail.com 
into the material up to a point (maximum depth dose), more secondary electrons are set in motion by secondary particles. Due to the having finite path lengths, number of electrons reaches a maximum at a particular depth and begin to decrease because the beam is attenuated as the depth increases. At this particular depth, the number of generated electrons is equal with the number of electrons which are stopped and deposited their energy. It is said that the charged particle equilibrium (CPE) exists at this point. In the near surface region, the required conditions of $\mathrm{CPE}$ are not achieved and even the dose gradient is quite high. Lack of CPE and being a high dose gradient region make the surface dosimetry complicated.[1]

The dose determination of a problematic region such as buildup region, specific dosimetric considerations should be taken. In treatment planning systems (TPSs), to get the accurate dose calculation depends on the selection of appropriate dosimetric systems and their correct use in dose measurements. For the precise beam modeling of the algorithms inside TPS, dose measurements required for modeling should be done with great precision. Especially in the problematic region as skin surface and buildup region, to avoid skin damage, to decide the thickness of the bolus material and radiotherapy treatment technique or the dose fractionation scheme, the amount of surface dose calculated by TPS should be known correctly. On the other hand, the dose calculation accuracy on the skin surface is a rather controversial issue. Unless they are well-modeled, the most commercial TPSs usually cannot calculate the surface dose accurately.[2] After a certain depth, the accuracy of the TPS can be considered satisfactory. In general, TPS algorithms tend to over or under-estimate surface dose. For example, Higgins et al.[3] reported that at the near surface, dose deviation between the calculated with Eclipse TPS and measured with $1 \mathrm{~mm}^{3}$ thermoluminescent dosimeters (TLD) chips is between $-12 \%$ and $10 \%$. In the anthropomorphic phantom study of Qi et al.,[4] it was reported that the Peacock system calculates the surface doses up to $8.5 \%$ higher than the measuring systems. Panettieri et al.[5] have investigated the calculation accuracy of Anisotropic Analytical Algorithm (AAA) and $\mathrm{PBC}$ algorithms between the surface and $2 \mathrm{~cm}$ depth and they compared the calculated data with Monte Carlo simulation. They showed that both algorithms underestimate the absorbed dose in the build-up region.

The selection of dosimetry systems for buildup region dose measurement is very vital. For a precise beam modeling in TPS, surface dose should be measured within at least $\pm 5 \%$ accuracy. Extrapolation chambers,
Attix chambers, TLDs, film dosimeters, and metal oxide semiconductor field effect transistors are the most common dosimetry systems used in surface dosimetry. For a more reliable dose measurement in buildup region, extrapolation chambers are suggested but these tools can be accessed only by a few institutions. Parallelplate ion chambers are good alternatives to the extrapolation chambers in surface dosimetry, but due to their over respond behaviors in high dose gradient region, some correction factors should be used (Gerbi and Khan's method).[6] When the appropriate corrections for polarity, obliquity, and in-scattering are applied to parallel-plate chamber measurements, similar results can be obtained to the extrapolation chambers considered as gold standard in the surface dose.[7] According to the study of Zhuang and Olch[2] the results of the Markus parallel plan ion chamber are compatible with the Monte Carlo simulation in the build-up region. Absolute dose differences were found within the $3 \%$.

Films are one of the passive detectors used in surface dosimetry. Gafchromic films are commonly used in radiotherapy dosimetry today because of their practical usage. They are insensitive to light, independent from dose fraction, dose rate and energy, do not require bathing as in radiographic films, and have a high spatial resolution. Because of their water equivalent feature, they are frequently preferred in in vivo dosimetry (IVD).

IVD is one the quality control method in radiotherapy. This method allows the measurement of the dose that reaches the patient during treatment and therefore it can be seen as the most reliable quality control method in radiotherapy. The dose control of the treatment is achieved by placing the detectors in natural body cavities or on the patient skin. Gafchromic films can be cut into small pieces, [8] give the users two-dimensional map of surface dose, and can record radiation permanently. Due to these favorable features, it is frequently preferred in IVD applications for skin dose measurements. Cao et al.[9] reported that superficial dose measurements obtained by Monte Carlo simulation are compatible with multilayer Gafchromic film results. In superficial region, they show a good agreement by the mean difference $<1 \%$.

The uncertainties and error probabilities that may arise in the progress of radiotherapy techniques can also increase. Despite the possible uncertainties that may derive from the characteristics of the modern radiotherapy treatment techniques, determining the dose applied to the target volume will be useful in controlling the accuracy of these techniques.[10] The comparison of the surface doses calculated in the TPSs and 
measured with different dosimetric systems is one of the techniques in the quality control programs of treatment plans. For this reason, surface dose responses of TPSs used in clinics and dosimetric systems used in patient surface dosimetry should be controlled by surface dosimetry systems which are accepted as reference.

In this study, the calculation accuracy of Acuros $\mathrm{XB}$ (AXB) and AAA v15.1algorithms is investigated and compared at the buildup region with parallel-plate ionization chamber and EBT3 films in different field sizes for $6 \mathrm{MV}$ photon energy. The behavior of the Gafchromic EBT3 film in surface region was checked by Markus parallel-plate ion chamber and after that the calculated results were compared with film. It was aimed that to determine the surface dose differences of the calculated by different algorithms and measured by film and to consider it during the quality control of the patient plans with IVD.

\section{Materials and Methods}

\section{Phantom and Parallel-Plate Ion Chamber Dose Mea- surements}

Varian Trilogy linear accelerator (Varian, Palo Alto, CA) equipped with a Millennium 120-leaf MLC was used in this study. All near surface central axis depth dose measurements were performed on the 6-MV photon beam along the central axis with open field sizes of $5 \times 5,10 \times 10$, and $20 \times 20 \mathrm{~cm}^{2}$ at a constant source-to-surface distance of $100 \mathrm{~cm}$. Since the measurements to be taken include problematic dose regions, SSD has not been changed to provide the necessary sensitivity for distance when solid water phantom is added on the plate to be taken at different depths. Then, source to skin distance correction for $\mathrm{SSD}=100 \mathrm{~cm}$ was applied to the results for each dosimeter. The measurements were performed in $40 \times 40$ $\mathrm{cm}^{2}$ solid water equivalent phantom slabs (SP34, PTW Freiburg, Freiburg, Germany) and the slab thickness varied from 1 to $10 \mathrm{~mm}$. The physical density of solid water phantom is $1.045 \mathrm{~g} / \mathrm{cm}^{-3}$. We obtained the central axis depth dose in the near surface region using the parallel-plate ionization chamber (Markus 23392, PTWFreiburg) and the EBT3 Gafchromic film (International Specialty Product, NJ, US). To acquire the average values, measurements were repeated 3 times.

The penetration range of the ion beam in a material is usually characterized by water equivalent thickness (WET). WET measures the amount of liquid water thickness that can stop the ion beam, as does a material of a certain thickness.[1] WET is calculated by taking into account the density of RW3 water equiva- lent phantom and the effective measurement depths of each dosimeter systems. If the dose responses of different dosimetry systems are to be compared at the same depth, the WET values must be known. Interpolation or extrapolation methods can also be used to obtain doses for different dosimeters at specific depths. WET values were calculated according to the effective measurement points of the dosimeters and hereby, depths corresponding to the read doses were obtained for all dosimetry systems. Interpolation and extrapolation methods were used to find the dose values at the same depths.

The recommendation of Commission on Radiation Units and Measurements and the International Commission on Radiological Protection about skin depth for surface dosimetry is $0.07 \mathrm{~mm} 8$ since this depth is generally corresponds to the interface between the dermis and epidermis layers of the skin. In our study, the comparisons were made at this recommended depth and $1,2,3,4$, and $5 \mathrm{~mm}$ which are accepted as near surface region.

In our study, one of the dosimeters used in the surface dose measurements is Markus parallel-plate ion chamber. The effective measurement point of Markus ion chamber is $0.023 \mathrm{~mm}$ which corresponds to the inner surface of the proximal collecting plate. The central axis near surface depth dose measurements were performed for $6 \mathrm{MV}$ photon beams, at a phantom depth of $0,1,2,3,4$, and $5 \mathrm{~mm}$ and $100 \mathrm{~cm}$ fixed SSD. Three readings were taken using the Unidos Webline electrometer (PTW Freiburg, Germany). The readings were taken for both positive and negative voltage $( \pm 300 \mathrm{~V})$ to take into account the polarity effects. Hereby the polarity correction factor was found and applies to readings. SSD correction factors were applied to the results. $100 \mathrm{MU}$ was delivered for each measurement and the results were normalized to $15 \mathrm{~mm}$ which is accepted as the maximum dose depth of $6 \mathrm{MV}$ photon beams.

Some electrons scattered from the side walls of fixed-separation parallel-plate ion chamber and in the buildup region they mainly contribute to the charge and these results to get over response dose by parallelplate ion chamber. Velkley et al.,[11] first introduced a correction factor for this perturbation effect. Gerbi and Khan improved the previous formula by including some parameters about parallel-plane ion chamber. The correction equation of GK (Gerbi and Khan) method [6] was given by Eqs. (1) and (2) below:

$$
\begin{aligned}
& P^{\prime}(d)=P(d)-\xi(E, 0) \ell e^{(-\alpha(d / d \max ))} \\
& (E, 0)=[-1.666+(1.982 I R)] \times(C-15.8)(\% / \mathrm{mm})
\end{aligned}
$$


Where P (d) is corrected and P'(d) is uncorrected percentage depth dose at "d" depth. $(E, 0)$ is the energy dependent chamber factor that indicates the over response in percent per $\mathrm{mm}$ of chamber plate separation at the phantom surface; $\ell$ is the plate separation and it is $2 \mathrm{~mm}$ for PTW Markus ion chamber; $\mathrm{d}$ and $\mathrm{d}_{\max }$ represent the depth of the chamber front window and maximum dose depth respectively. IR is the ionization ratio that measured at 10 and $20 \mathrm{~cm}$ depths, $10 \times 10 \mathrm{~cm}^{2}$ field size at SSD $100 \mathrm{~cm}$. For $6 \mathrm{MV}$ photon beam, IR is 0.6709 . $\mathrm{C}$ is the distance between the collector edge and side wall, and it is regarded as $0.35 \mathrm{~mm}$ for PTW Markus ion chamber. $\alpha$ is a constant and equal to 5.5. All factors were placed in equations above and the over response correction factors were obtained. Thus, Grebi and Khan's overdose correction method[6] was applied to our PDDs.

\section{Film Dose Measurements}

In the present study, we used Gafchromic EBT3 films (International Specialty Product, NJ, US) from the same batch which has a single active layer of approximately $30 \mu \mathrm{m}$ thickness. Gafchromic EBT3 film has a $30 \mu \mathrm{m}$ thickness of single active layer which is between $125 \mu \mathrm{m}$ thick of transparent polyester sheets. The effective measurement point of EBT3 film was assumed at the depth of $0.153 \mathrm{~mm}$.[12] A calibration curve was created to accurately evaluate the irradiated films and the irradiated films were calibrated according to this curve. To generate film calibration curve, films were cut into small pieces. They were placed at $5 \mathrm{~cm}$ depth which was defined as the depth of calibration for $6 \mathrm{MV}$ photon beams. All films were irradiated within the dose ranges of $0-800 \mathrm{cGy}$ at the field of $10 \times 10 \mathrm{~cm}^{2}$. One of the films was released for the background without irradiation. After $24 \mathrm{~h}$, all the films were scanned by Epson Expression 10000XL scanner. Irradiated films were analyzed in Image J program and separated into three different channels (red, blue, and green channels). In Gafchromic films, the largest contrast difference is seen in red channel therefore red color was selected for dose reading. The film calibration program in Mephysto mcc software (PTW Mephysto $\mathrm{mc}^{2}$, PTW, Freiburg, Germany) was used to create calibration curve. Using this program, the optical densities in the center of scanned films was read. A calibration curve was created based on the permeability values corresponding to the dose values.

Film measurements were performed at the same setup conditions as the ion chamber measurements. Due to the different effective measurement point of film, the WET values corresponding to the measured doses were calculated carefully.

\section{TPS Dose Calculations}

Varian Eclipse TPS (Varian Headquarters, Palo Alto, California, USA) which has AAA 15.1 versions and AXB algorithms was used for TPS dose calculations. In TPS, a water phantom size of $40 \times 40 \times 10 \mathrm{~cm}^{3}$ was formed automatically and beams were set on it in the same field sizes and gantry angles as described in parallel-plate ion chamber and Gafchromic film measurements. The fraction dose for each plan was set at $100 \mathrm{MU}$, the doses which the dosimetry systems were irradiated also. All the plans were calculated with different algorithms for the same setup conditions. The calculation grid size of 1 $\mathrm{mm}$ was used for all calculation algorithms. Doses were read at the depth of effective measurement of film and ion chamber from the TPS. The calculated PDDs were normalized the dose at $1.5 \mathrm{~cm}$ depth for each field sizes.

\section{Results}

The doses in the near surface for $6 \mathrm{MV}$ photon beams were measured with Gafchromic EBT3 film and Markus parallel-plate ion chamber for the square field sizes of $5 \times 5,10 \times 10$ and $20 \times 20 \mathrm{~cm}^{2}$. The effective measurement point of both dosimetry systems was noted and to obtain doses at $0.07,1,2,3,4$, and 5-mm depth for each dosimetry, interpolation, and extrapolation were made using four-order polynomial functions. The percentage doses at the surface and buildup regions for different field sizes measured using the Markus parallel plane ion chamber are given in Table 1 .

\begin{tabular}{|c|c|c|c|c|}
\hline \multirow[t]{2}{*}{ Table 1} & \multicolumn{4}{|c|}{$\begin{array}{l}\text { Percentage depth doses (PDDs) obtained with } \\
\text { a Markus parallel plane ion chamber in a water } \\
\text { equivalent RW3 phantom using } 6 \mathrm{MV} \text { photon } \\
\text { beams for different field sizes at } S S D=100 \mathrm{~cm} \text {. }\end{array}$} \\
\hline & & & 'DD (\%) & \\
\hline $\begin{array}{l}\text { Phantom } \\
\text { Depth (mm) }\end{array}$ & $\begin{array}{l}\text { WET } \\
(\mathrm{mm})\end{array}$ & $5 \times 5 \mathrm{~cm}^{2}$ & $10 \times 10 \mathrm{~cm}^{2}$ & $20 \times 20 \mathrm{~cm}^{2}$ \\
\hline 0 & 0.023 & 10.81 & 16.61 & 28.06 \\
\hline 0.067 & 0.07 & 14.00 & 19.69 & 30.87 \\
\hline 1 & 1.068 & 38.74 & 43.44 & 52.48 \\
\hline 2 & 2.113 & 57.12 & 60.98 & 68.19 \\
\hline 5 & 5.248 & 83.65 & 85.95 & 90.00 \\
\hline 10 & 10.473 & 97.77 & 98.37 & 99.24 \\
\hline 15 & 15.698 & 100.00 & 100.00 & 100.00 \\
\hline
\end{tabular}

PDD: Percentage depth dose; WET: Water equivalent thickness 
The interpolated and extrapolated skin dose values at $0.07 \mathrm{~mm}$ for Markus ion chamber and EBT3 film are $14 \%$ and $12.79 \%$ for $5 \times 5 \mathrm{~cm}^{2}$; and $30.87 \%$ and $27.07 \%$ for $20 \times 20 \mathrm{~cm}^{2}$, respectively. The PDD curve comparison for the measurement for a $10 \times 10 \mathrm{~cm}^{2}$ field size is shown in Figure 1.

Markus parallel plane ion chamber can be considered as a reference dosimetry in surface dosimetry. On the other hand, because of their physical characteristics they cannot be used in IVD. Its characteristics such as low spectral sensitivity and high spatial resolution make the film an appropriate dosimeter system in surface and buildup region dosimetry. In Figure 1, it is seen that in the near surface EBT3 film shows close results with the Markus ion chamber for all field sizes and depths.

Each dosimeter has a different effective measurement depth. To obtain percentage depth dose values of film and TPS at the same depths, WET of the systems should be considered. The WET values belong to Gafchromic EBT 3 Film are given in Table 1 and they were used to obtain the PDD values from TPS for the same depths. The PDD results at $0.07 \mathrm{~mm}$ for $5 \times 5$, $10 \times 10$, and $20 \times 20 \mathrm{~cm}^{2}$ field sizes acquired by TPS algorithms and Film are shown in Figure 2.

As shown from Figure 2, for all field sizes AAA v15.1 shows highest results. For $10 \times 10$ and $20 \times 20 \mathrm{~cm}^{2}$, surface depth doses calculated by Acuros algorithm and measured by Gafchromic EBT 3 film are nearly similar. On the other hand, as shown in Figure 3, for all field sizes by increasing depth, all algorithms and film depth dose values were approaching to each other.

\section{Discussion}

Although the surface dose measurement is a challenging issue, it is the best reliable QA method to control the dose that reaches the patient during treatment which is called IVD. One of the most preferable IVD methods is to control of the skin dose by placing the dosimeter on the patient skin. By this method, the quality of the patient treatment plans can be checked in the most correct way.

To measure doses of buildup region accurately, dosimetry preference is very crucial. As known from previous studies, extrapolation chambers are the recommended dosimeters for surface dosimetry.[11] Nevertheless, Markus parallel plane ion chamber can be a good alternative to extrapolation chambers. Although Markus parallel plate ion chambers are preferred for surface dose measurements, they are not suitable for IVD due to their physical properties. Therefore, in this study,

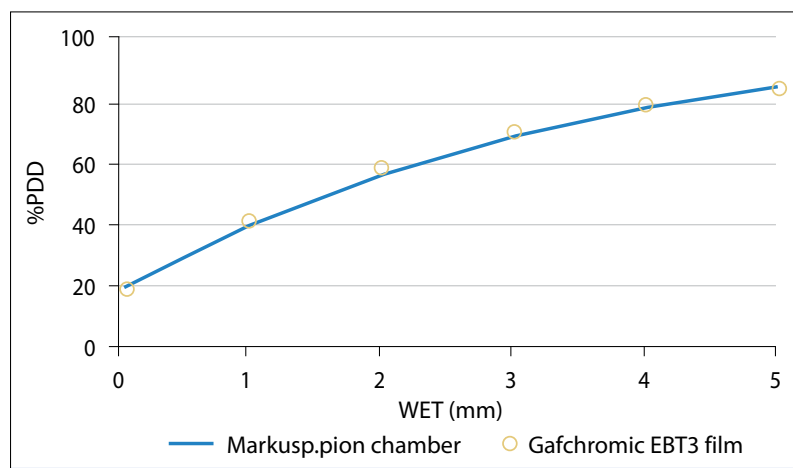

Fig. 1. Percentage depth doses (PDDs) at surface and buildup regions for $6 \mathrm{MV}$ photon beams using a Markus parallel plate ion chamber and Gafchromic EBT3 film.

PDD: Percentage depth dose, WET: Water equivalent thickness.

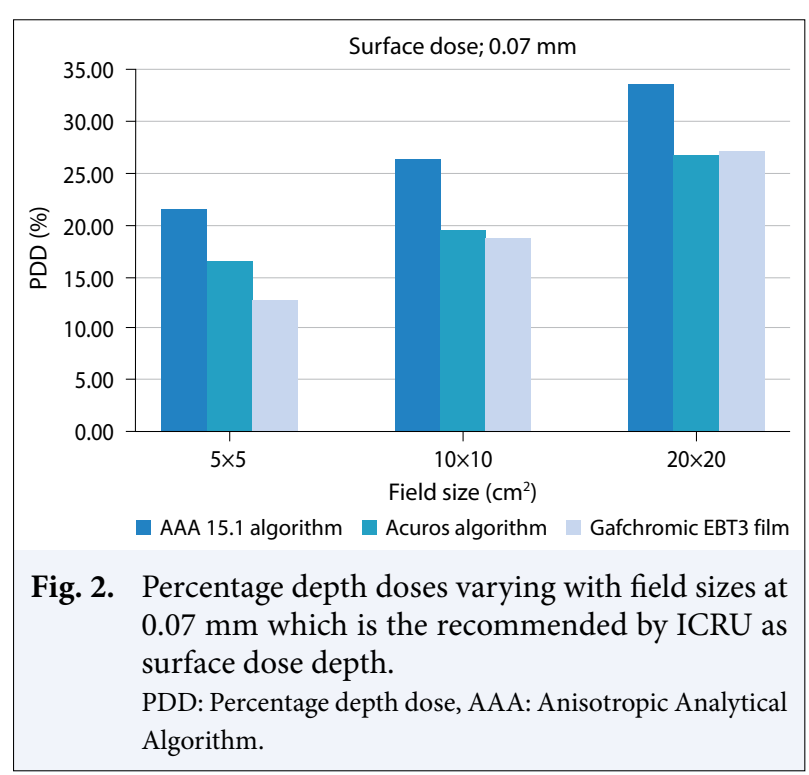

it was only used to determine the accuracy of the film in surface dose measurements as a reference dosimetry.

Radiochromic films are good options for surface and buildup region dose measurements and are suitable for IVD. In our study, Markus parallel plate ion chamber was used as a reference dosimetry and buildup region depth dose values were obtained. The results were compared by Gafchromic EBT3 films. For $10 \times 10 \mathrm{~cm}^{2}$ field size, surface doses at 0.07 $\mathrm{mm}$ with the Markus parallel-plate ion chamber and Gafchromic EBT3 film were found $16.61 \%$ and $18.8 \%$, respectively. Close results show that also film can be used as a reference dosimetry system for surface dosimetry and it was used to control the accuracy of TPS algorithms in our study. 


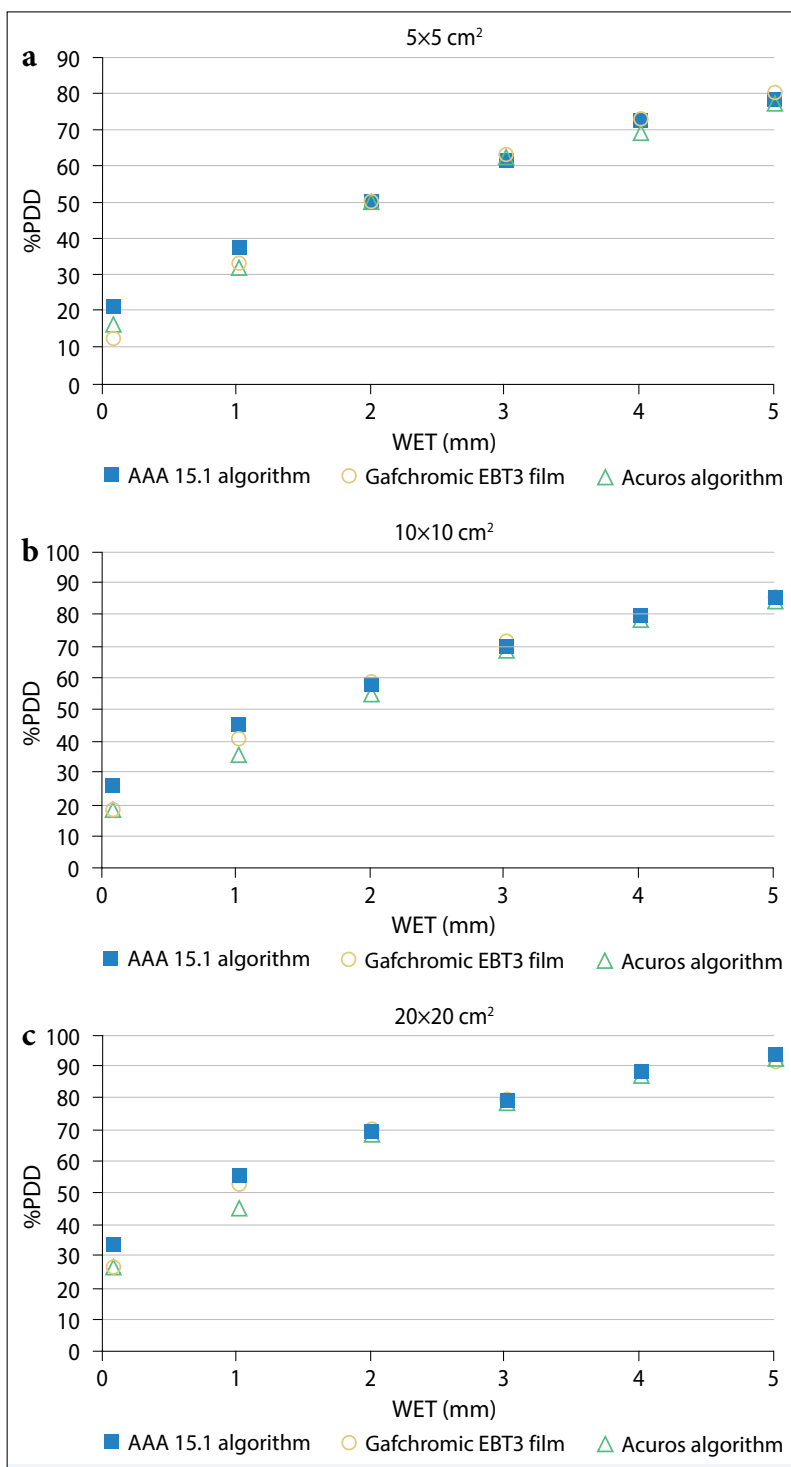

Fig. 3. Percentage depth doses (PDDs) for $6 \mathrm{MV}$ photon beams with different sizes at $100 \mathrm{~cm}$ fixed SSD. The doses at $0,1,2,3,4$, and $5 \mathrm{~mm}$ depths were obtained by Gafchromic EBT 3 film and Eclipse TPS with AAA v15.1 and Acuros algorithms, and the following field sizes were investigated: $5 \times 5$ $\mathrm{cm}^{2}$ (a), $10 \times 10 \mathrm{~cm}^{2}$ (b), and $20 \times 20 \mathrm{~cm}^{2}$ (c). PDD: Percentage depth dose, AAA: Anisotropic Analytical Algorithm, WET: Water equivalent thickness.

The harder it is to measure the skin dose correctly, the harder it is to calculate it through TPS. Algorithms inside TPS cannot calculate buildup region doses correctly. In our study, the film measurements were compared by TPS results, calculated and measured surface dose differences were obtained. These differences obtained through the phantom will guide us while checking the patient treatment plans through IVD.
Bilge et al.[13] measured the surface dose with the Markus parallel plate ion chamber for 6 MV (Siemens Oncor Impression Plus linear accelerator) photon beams and the result was found to be $15.0 \%$ for a field size of $10 \times 10 \mathrm{~cm}^{2}$. They also used EBT model radiochromic film and found the surface dose at the depth of $0 \mathrm{~mm} 20.0 \pm 2 \%$ for a field size of $10 \times 10 \mathrm{~cm}^{2}$. The surface dose with Gafchromic EBT3 film was found to be $20.4 \%$ at the same depth and field size.

Qi et al.[4] investigated the dose at the WET of Gafchromic EBT3 film $0.153 \mathrm{~mm}$ which equals the depth of $0 \mathrm{~mm}$ in the phantom, for the same field size and the result was found $23.5 \%$. Our study is consistent with the previous studies.

Cao et al.[9] investigate the superficial dose accuracy of different algorithms comparing by Monte Carlo algorithm and film measurements. Surface and buildup region doses were measured by the group. They showed that AAA algorithm overestimate skin dose near 4.07\% when compared by Monte Carlo simulation. Nevertheless within 2-15 mm depth, results were compatible by Monte Carlo.

Cho et al.[14] studied about skin dose measurements for lung stereotactic body radiotherapy. Measurements were taken by GafChromic EBT2 film, Attix chamber and compared to near surface skin doses predicted by Eclipse TPS using the AAA algorithm for a $6 \mathrm{MV}$ photon beam. For $10 \times 10 \mathrm{~cm}^{2}$ field size, surface doses at $0 \mathrm{~mm}$ with the Attix chamber, GafChromic EBT2, and AAA algorithm were found 16\%, 23.9\%, and $40 \%$, respectively. Their results show that AAA algorithm overestimates the surface dose at the top of the phantom (nominal $0 \mathrm{~mm}$ depth) compared to the measured data by Attix chamber and EBT2 film.

In our study, the calculated results obtained by AAA v15.1 and Acuros algorithms were compared by Gafchromic EBT3 film. For $10 \times 10 \mathrm{~cm}^{2}$ field size, surface doses at $0.07 \mathrm{~mm}$ with the AAA v15.1, Acuros algorithms and Gafchromic EBT3 were found 26.25\%, $19.5 \%$, and $18.8 \%$, respectively. It was seen that for all field sizes AAA v15.1 result was much higher than film and Acuros algorithm. In addition to that, surface doses obtained by Markus chamber and Gafchromic EBT3 film at $0 \mathrm{~mm}$ depth were $16.61 \%$ and $20.40 \%$ for $10 \times 10 \mathrm{~cm}^{2}$ field size. The results present coherency with the study of Cho et al.[14]

According to the many researches, AXB algorithm can calculate the dose accurately in regions with complex geometries and heterogeneities.[15,16] The studies about AXB algorithm were usually to investigate the calculation capability of the algorithm in inhomoge- 
neous mediums. There is not enough study about their behaviors in the surface and buildup region. Alhakeem et al.[17] studied about the performance of different dosimetric methods in inhomogeneous phantoms. They used two versions of AXB and AAA algorithms, EBT2 film and MOSkin dosimeters and compared the results by Monte Carlo calculations. It was seen that the newer version of AXB (v11.0.31) algorithm and film results were closer to MC calculation than AAA algorithm. In the near surface, AXB (v11.31) and Gafchromic EBT2 results were seen similar. From the near surface to $5 \mathrm{~mm}$, Gafchromic EBT2 film showed lower results than all algorithms; however, after $5 \mathrm{~mm}$ all the values were approaching each other. In our results, for all field sizes, in the buildup region, the results obtained by AAA were found higher than AXB and Gafchromic EBT3 results while AXB and Gafchromic EBT3 results were close to each other. After $2 \mathrm{~mm}$ depth, all the calculated and measured depth doses were close to each other.

\section{Conclusion}

The accuracy of algorithms used to prepare the treatment plans is an important issue. TPS has difficulties in calculating, such as in heterogeneous or buildup regions. Especially in the treatment of superficial cancers or in the situation which the surface dose information is crucial, the behaviors of algorithms in the buildup region must be known precisely.

In clinics, to be sure of the correctness of surface and buildup region dose calculation, QA procedures must be performed for all algorithms implanted in TPS before using them for patient treatment planning. By this means, especially for treatment plans where surface dose information is important, the behavior of the algorithms in the surface region can be predicted. It will be valuable to know the calculation capabilities of the algorithms in the surface region in the quality control of patient treatment plans with IVD.

Gafchromic EBT3 film is a useful dosimetry system for IVD. Since it exhibits the similar results by Markus parallel plate ion chamber in the surface and buildup region, Gafchromic EBT3 film can be a preferable dosimeter in QA programs for skin dose control of the patients.

Peer-review: Externally peer-reviewed.

Conflict of Interest: All authors declared no conflict of interest.
Ethics Committee Approval: The study was approved by the Academic Coordination Community of Istanbul University Institute of Oncology Ethics Committee (No: B.30.2.ÍST.0.53.00.00/911, Date: 28/03/2013).

Financial Support: None declared.

Authorship contributions: Concept - N.D.K., C.K.A.; Design - N.D.K.; Supervision - N.D.K.; Funding - None; Materials - N.D.K., C.K.A.; Data collection and/or processing N.D.K., C.K.A.; Data analysis and/or interpretation - N.D.K., C.K.A.; Literature search - N.D.K.; Writing - N.D.K.; Critical review - N.D.K., C.K.A.

\section{References}

1. de Vera P, Abril I, Garcia-Molina R. Water equivalent properties of materials commonly used in proton dosimetry. Appl Radiat Isot 2014;83(Pt B):122-7.

2. Zhuang AH, Olch AJ. Validation of OSLD and a treatment planning system for surface dose determination in IMRT treatments. Med Phys 2014;41(8):081720.

3. Higgins PD, Han EY, Yuan JL, Hui S, Lee CK. Evaluation of surface and superficial dose for head and neck treatments using conventional or intensity-modulated techniques. Phys Med Bio 2007;52(4):1135-46.

4. Qi ZY, Deng XW, Huang SM, Zhang L, He ZC, Li XA, et al. In vivo verification of superficial dose for head and neck treatments using intensity-modulated techniques. Med Phys 2009;36(1):59-70.

5. Panettieri V, Barsoum P, Westermark M, Brualla L, Lax I. AAA and PBC calculation accuracy in the surface build-up region in tangential beam treatments. Phantom and breast case study with the Monte Carlo code PENELOPE. Radiother Oncol 2009;93(1):94-101.

6. Gerbi BJ, Khan FM. Measurement of dose in the buildup region using fixed-separation plane-parallel ionization chambers. Med Phys 1990;17(1):17-26.

7. Tatsiana AR, Patrick H. Surface dose measurements with commonly used detectors: A consistent thickness correction. J Appl Clin Med Phys 2015;16(5):358-66.

8. Devic S, Seuntjens J, Abdel-Rahman W, Evans M, Olivares, M, Podgorsak EB, et al. Accurate skin dose measurement using radiochromic film in clinical applications. Med Phys 2006;33(4):1116-24.

9. Cao Y, Yang X, Yang Z, Qiu X, Lv Z, Lei M, et al. Superficial dose evaluation of four dose calculation algorithms. Radiat Phys Chem 2017;137(1):23-8.

10. Scarantino CH, Prestidge BR, Anscher MS, Ferree $\mathrm{CR}$, Kearns WT, et al. The observed variance between predicted and measured radiation dose in breast and prostate patients utilizing an in vivo dosimeter. Int J Radiat Oncol Biol Phys 2008;72(2):597-604.

11. Velkley DE, Manson DJ, Purdy JA, Oliver GD Jr. Build- 
up region of megavoltage photon radiation sources. Med Phys 1975;2(1):14-9.

12. Van Battum LJ, Hoffmans D, Piersma H, Heukelom S. Accurate dosimetry with GafChromic EBT film of a 6 MV photon beam in water: What level is achievable? Med Phys 2008;35(2):704-16.

13. Bilge H, Çakır A, Okutan M, Acar H. Surface dose measurements with GafChromic EBT film for 6 and 18MV photon beams. Phys Med 2009;25(2):101-4.

14. Gwi AC, Ralston A, Mo TM, Martin D, Pickard S, Kim $\mathrm{JH}$, et al. In vivo and phantom measurements versus Eclipse TPS prediction of near surface dose for SBRT treatments. J Phys 2014;489:012008.

15. Bush K, Gagne IM, Zavgorodni S, Ansbacher W, Beckham W. Dosimetric validation of Acuros XB with Monte Carlo methods for photon dose calculations. Med Phys 2011;38(4):2208-21.

16. Lloyd S, Ansbacher W. Evaluation of an analytic linear Boltzmann transport equation solver for high-density inhomogeneities. Med Phys 2013;40(1):011707.

17. Alhakeem EA, Al Shaikh S, Rosenfeld AB, Zavgorodni SF. Comparative evaluation of modern dosimetry techniques near low and high-density heterogeneities. J Appl Clin Med Phys 2015;16(5):142-58. 